\title{
Boronic acid with high oxidative stability and utility in biological contexts
}

\author{
Brian J. Graham ${ }^{a}$, lan W. Windsor ${ }^{a}$, Brian Gold ${ }^{b}$, and Ronald T. Raines ${ }^{* 1}$ \\ Department of Chemistry, Massachusetts Institute of Technology, Cambridge, MA 02139
}

\begin{abstract}
Author Contributions
aEqual contributions Albuquerque, NM 87131

ORCID

B.J.G. 0000-0001-9985-3553

I.W.W. 0000-0002-6289-6928

B.G. $0000-0002-3534-1329$

R.T.R. 0000-0001-7164-1719
\end{abstract}

All authors designed research; B.J.G. and I.W.W. performed experimental research; B.G. and I.W.W. performed computational research; all authors analyzed data and wrote the paper.

${ }^{b}$ Current address: Department of Chemistry and Chemical Biology, University of New Mexico,

${ }^{1}$ To whom correspondence may be addressed. Email: rtraines@mit.edu 


\author{
Abstract \\ Despite their desirable attributes, boronic acids have had a minimal impact in biological \\ contexts. A significant problem has been their oxidative instability. At physiological $\mathrm{pH}$, \\ phenylboronic acid and its boronate esters are oxidized by reactive oxygen species at rates \\ comparable to those of thiols. After considering the mechanism and kinetics of the oxidation \\ reaction, we reasoned that diminishing electron density on boron could enhance oxidative \\ stability. We found that a boralactone, in which a carboxyl group serves as an intramolecular \\ ligand for the boron, increases stability by $10^{4}$-fold. Computational analyses revealed that the \\ resistance to oxidation arises from diminished stabilization of the p orbital of boron that develops \\ in the rate-limiting transition state of the oxidation reaction. Like simple boronic acids and \\ boronate esters, a boralactone binds covalently and reversibly to 1,2-diols, such as those in \\ saccharides. The kinetic stability of its complexes is, however, at least 20 -fold greater. A \\ boralactone also binds covalently to a serine side chain in a protein. These attributes confer \\ unprecedented utility upon boralactones in the realms of chemical biology and medicinal \\ chemistry.
}




\section{Significance Statement}

Like thiols, boronic acids are unstable to oxidation in biological contexts, limiting their utility. We discovered that the rate-limiting step in the oxidation of a boronic acid can be slowed by the installation of a pendant carboxyl group that is a ligand to the boron atom. The ensuing boronic acid is ten thousand-fold more resistant to oxidation. Experimental and computational analyses reveal that the orientational constraints and electronic effects imposed by the carboxyl group act as intended, slowing the rate-limiting step during oxidation. The new boronic acid retains the useful attributes of simple boronic acids, such as forming reversible covalent bonds with saccharides and a protein, and could be useful in chemical biology, medicinal chemistry, and allied fields. 
The modern pharmacopeia is composed of only a handful of elements. Built on hydrocarbon scaffolds (1), nearly all drugs contain nitrogen and oxygen, and many contain fluorine and sulfur (2). A surprising omission from this list is the fifth element in the periodic table: boron $(3,4)$. Since bortezomib received regulatory approval in 2003, only four additional boron-containing drugs have demonstrated clinical utility (Fig. 1A). Each is a boronic acid or ester.

Bortezomib is a boronic acid and ixazomib citrate hydrolyzes to one in aqueous solution (5). Other boron-containing drugs feature cyclic esters. The cyclic ester formed spontaneously from 2-hydroxymethylphenylboronic acid (2-HMPBA) is known as "benzoxaborole" and has received much attention due to its enhanced affinity for saccharides at physiological $\mathrm{pH}(6-8)$. This scaffold is present in the antifungal drug tavaborole and the antidermatitis drug crisaborole (9). Vaborbactam, which contains an analogous six-membered ring, is an efficacious $\beta$-lactamase inhibitor (10). Neuropathy has been associated with the use of bortezomib but not other boronic acids, which have minimal toxicity (11).

The boron atom in a boronic acid (or ester) is isoelectronic with the carbon atom of a carbocation. Both are $\mathrm{sp}^{2}$ hybridized, have an empty $\mathrm{p}$ orbital, and adopt a trigonal planar geometry. In contrast to a carbocation, however, the weak Lewis acidity of a boronic acid allows for the reversible formation of covalent bonds. This attribute has enabled boronic acids to achieve extraordinary utility in synthetic organic chemistry and molecular recognition (12-22). Boronic acids are, however, susceptible to oxidative damage. That deficiency is readily controllable in a chemistry laboratory but not in a physiological environment.

In a boronic acid, the empty p orbital of boron is prone to attack by nucleophilic species such as the oxygen atom of a reactive oxygen species (ROS). The subsequent migration of carbon from boron to that oxygen leads to a labile boric ester, which undergoes rapid hydrolysis (Fig. 
$1 B)$. This oxidative deboronation converts the boronic acid into an alcohol and boric acid (23, 24).

We sought a means to increase the utility of boron in biological contexts by deterring the oxidation of boronic acids. The rate-limiting step in the oxidation of boronic acid is likely to be the migration of carbon from boron to oxygen: a 1,2-shift (Fig. 1B). In that step, the boron becomes more electron-deficient. We reasoned that depriving the boron of electron density might slow the 1,2-shift. A subtle means to do so would be to replace the alkoxide of a boronate ester with a carboxylate group. We find that the ensuing mixed anhydrides between a boronic acid and carboxylic acid are remarkable in their chemical attributes and biological utility.

\section{Results}

$\mathrm{p} K_{\mathrm{a}}$ Values and Crystal Structure of a Boronic Acid-Carboxylic Acid Mixed Anhydride.

We began by characterizing the protonation state in water of a simple boronic acid that is capable of forming a mixed anhydride: 2-carboxyphenylboronic acid (2-CPBA) (25-27). Notably, boronic acids are Lewis acids that ionize after formation of a complex with water or another protic solvent. A potentiometric $\mathrm{pH}$-titration revealed that the $\mathrm{p} K_{\mathrm{a}}$ values of 2-CPBA are extreme. ${ }^{11} \mathrm{~B}-\mathrm{NMR}$ titration experiments showed that a single species is dominant from $\mathrm{pH} 0.9-$ 12 and has a chemical shift consistent with the monoanion. 2-CPBA was converted to the dianion at high $\mathrm{pH}$, affording a $\mathrm{p} K_{\mathrm{a} 2}$ value of 13.0 (SI Appendix, Fig. S1). No ${ }^{11} \mathrm{~B}$ chemical shift consistent with a neutral species was observed at any $\mathrm{pH}$ value, even in $6 \mathrm{M} \mathrm{HCl}$.

To provide further insight, we crystallized 2-CPBA at low $\mathrm{pH}$ and used $\mathrm{X}$-ray diffraction analysis to determine its structure (Tables S1-S7). Crystalline 2-CPBA was a zwitterion with a tetrahedral boron atom and protonated hydroxy group (Fig. 2A; SI Appendix, Fig. S2). Thus, the boralactone ring of the anhydride was maintained rather than dissociated into boronic acid and 
carboxylic acid groups. We conclude that the predominant form of 2-CPBA in water is the monoanionic benzoxaborolone. The monoanion also predominates in DMSO. In $\mathrm{CD}_{3} \mathrm{OD}$, the ${ }^{11} \mathrm{~B}-\mathrm{NMR}$ peak shifts to that of a more neutral species, but one that is still more anionic than a typical phenylboronic acid (SI Appendix, Fig. S1). Likewise, ${ }^{11} \mathrm{~B}-\mathrm{NMR}$ analysis of the homologated 2-carboxymethylphenylboronic acid (2-CMPBA) showed chemical shifts similar to those of 2-CPBA even in a highly acidic solution, again indicative of a tetrahedral anionic boron.

Boronic Acid-Carboxylic Acid Mixed Anhydrides Resist Oxidation. Next, we assembled a panel of representative boronic acids for comparison to 2-CPBA (Fig. 2A). The homologated six-membered ring of benzoxaborininone (BON), which derives from 2-CMPBA, deserves attention. The benzoxaborole moiety (BL) was embodied by 2-hydroxymethylphenylboronic acid (2-HMPBA). Its homologated analog, benzoxaborinine (BN), has been reported to be stable in water (28) and was derived from 2-hydroxyethylphenylboronic acid (2-HEPBA). Phenylboronic acid (PBA) has been studied extensively (29-36) and was used here as a reference.

Hydrogen peroxide is the major reactive oxygen species in humans $(37,38)$ and thus provides a physiologically relevant oxidative deboronation pathway in vitro. The oxidation of phenylboronic acids by aqueous hydrogen peroxide yields a phenol and boric acid (Fig. 1B). As the phenol product exhibits a significant blue-shift in UV absorbance relative to the boronic acid starting material, we were able to monitor oxidation with a continuous assay based on UV absorbance (SI Appendix, Table S8 and Fig. S3) (39). We found that the oxidation rates were first-order with respect to hydrogen peroxide and boronic acid (SI Appendix, Fig. S4). The ensuing second-order rate constants varied with pH (SI Appendix, Table S9) and were halfmaximal for PBA and $\mathrm{BL}$ when $\mathrm{pH}=\mathrm{p} K_{\mathrm{a}}$. We found that oxidation rates increase in a 
$\mathrm{pH}$-dependent manner before and after $\mathrm{pH}$-independent regimes (Fig. $2 B$ ), with a high correlation to the predicted speciation between trigonal and tetrahedral boron based on the $\mathrm{p} K_{\mathrm{a}}$ values. These data are consistent with the existence of two $\mathrm{pH}$-independent rate constants, $k_{1}$ and $k_{2}$.

A comparison of boronic acids to thiols is pertinent. The sulfhydryl group is the most abundant redox-active functional group in physiological contexts. The oxidation of sulfhydryl groups to disulfides or sulfenic acids is readily reversible by either enzymic catalysts or reaction with small-molecule thiols, such as reduced glutathione. In contrast, the oxidation of boronic acids to alcohols is irreversible (Fig. $1 B$ ). We found that the oxidation rates of PBA and BL at physiological $\mathrm{pH}$ are comparable to those of biological thiols (Table 1). In comparison, BOL is $10^{4}$-fold more stable.

In $\mathrm{BL}$ and $\mathrm{BOL}$, boron resides in a five-membered ring. We found that the additional flexibility of a six-membered ring increased oxidation rates. For BN, the oxidation rates showed two regimes: at low $\mathrm{pH}$ the rates were similar to those of $\mathrm{BL}$, whereas at high $\mathrm{pH}$, the rates were similar to those of PBA. As in the context of a five-membered ring, depriving the boron of electron density stabilized a six-membered ring against oxidation. At physiological $\mathrm{pH}$, the oxidative stability of BON was greater than that of BL but less than that of BOL (Table 1 and Fig. 2B).

\section{$B \rightarrow O$ Shift Limits the Rate of Boronic Acid-Carboxylic Acid Mixed Anhydride}

Oxidation. To identify the rate-limiting step in the oxidation rate of BOL, we performed a kinetic competition experiment. Specifically, we employed a fluorogenic boronic acid, coumarin boronic acid (CBA), which like PBA oxidizes rapidly to form 7-hydroxycoumarin under conditions that leave BOL intact (Fig. 3A) (40). We found that the addition of excess BOL led to a concentration-dependent reduction in the rate of CBA oxidation (Fig. $3 B$ ). Because the rate of 
BOL oxidation is exceeding slow, BOL cannot be consuming hydrogen peroxide. Instead, BOL is sequestering hydrogen peroxide. This conclusion is consistent with ${ }^{1} \mathrm{H}-\mathrm{NMR}$ spectra acquired during the oxidation of BOL (SI Appendix, Fig. S5). These spectra revealed the accumulation of covalent intermediates that were not apparent during the oxidation of PBA or BL, and that are consistent with a tetrahedral complex with hydrogen peroxide anion. Significantly, too, the second-order rate constant for the complexation of ligands to boronic acids $\left(10^{3}-10^{5} \mathrm{M}^{-1} \mathrm{~s}^{-1}\right)(41$ 43) are $>10^{6}$-fold greater than that of BOL oxidation (Table 1). Thus, we conclude that the $\mathrm{B} \rightarrow \mathrm{O}$ shift of the intermediate rather than its formation limits the rate of BOL oxidation.

\section{Resistance of Boronic Acid-Carboxylic Acid Mixed Anhydrides to Oxidation Derives}

from Stereoelectronic Effects. We employed computational analyses to elucidate the physical origin of the oxidative stability of BOL. BOL and BON exist almost solely as monoanionic tetrahedral species in aqueous solution (Fig. 2A). Accordingly, we facilitated comparisons by performing all of our calculations on boronic acid monoanions. We used density functional theory calculations to model stationary points along the reaction coordinate for the oxidation of each boronic acid, enabling energetic comparisons with the $\mathrm{pH}$-independent rate constant, $k_{\text {monoanion. }}$ We began by modeling the tetrahedral intermediate formed with hydrogen peroxide and the boric acid ester products (SI Appendix, Tables S10 and S11, and Fig. S6). Then, we calculated transition-state structures for the oxidation reaction (Fig. 4), which replicated the results of similar systems (44). Optimized transition state structures show a concerted mechanism in which the 1,2-shift from boron to oxygen is concomitant with oxygen-oxygen bond cleavage, which agrees with previous mechanistic work $(30,32,45,46)$. The relative rate constants for oxidation calculated from these structures match exceptionally well with experimental values (Fig. 5 and SI Appendix, Table S12). 
Next, we turned to Natural Bonding Orbital (NBO) analysis (47) to reveal interactions that are responsible for the increased activation energies of $\mathrm{BL}, \mathrm{BON}$, and $\mathrm{BOL}$, relative to PBA. During the transformation from the tetrahedral boronate ester ( $\mathrm{sp}^{3}$ boron) to the planar, boric acid ester ( $\mathrm{sp}^{2}$ boron), the $\mathrm{C}-\mathrm{B}$ bond breaks and becomes a $\mathrm{C}-\mathrm{O}$ bond, leaving boron with a vacant $\mathrm{p}$ orbital. We found that the observed differences in reactivity stem from differential stabilization of the developing $\mathrm{p}$ orbital on boron $\left(\mathrm{p}_{\mathrm{B}}\right)$ by neighboring oxygen lone pairs $\left(\mathrm{n}_{\mathrm{O}}\right)$ in the transition state.

Without cyclic constraints, each hydroxy group of PBA stabilizes the developing p orbital on boron via a classical conjugative $\mathrm{n}_{\mathrm{O}} \rightarrow \mathrm{p}_{\mathrm{B}} \pi$-bonding interaction (Fig. $4 A$ ). These interactions are absent in the starting tetrahedral boronate (where a much weaker $n_{\mathrm{O}} \rightarrow \sigma^{*}{ }_{\mathrm{B}-\mathrm{C}}$ interaction of 8.0 $\mathrm{kcal} / \mathrm{mol}$ is present) but are worth $35.2 \mathrm{kcal} / \mathrm{mol}$ in the transition state.

The structure of the cyclic systems results in a "twisting" of the $n_{O, p}$ lone pairs out of conjugation with the $\mathrm{p}_{\mathrm{B}}$ orbital. This twisting decreases the $\mathrm{n}_{\mathrm{O}, \mathrm{p}} \rightarrow \mathrm{p}_{\mathrm{B}}$ interaction to $20.4 \mathrm{kcal} / \mathrm{mol}$ in the BL transition state (Fig. $4 B$ ). In addition to decreased $n_{O, p}-p_{B}$ overlap, the ability of the $\mathrm{n}_{\mathrm{O}, \mathrm{p}}$ orbital to donate electron density is decreased in boralactones $\mathrm{BON}$ and $\mathrm{BOL}$ by conjugation to the carbonyl $\pi$-system (i.e., ester resonance). This "pulling" decreases the $\mathrm{n}_{\mathrm{O}, \mathrm{p}} \rightarrow \mathrm{p}_{\mathrm{B}}$ interaction to $14.8 \mathrm{kcal} / \mathrm{mol}$ in the BON transition state (Fig. $4 C$ ). Most notably, the $\mathrm{n}_{\mathrm{O}, \mathrm{p}}$ and $\mathrm{p}_{\mathrm{B}}$ orbitals in the BOL transition state approach orthogonality, and the $\mathrm{n}_{\mathrm{O}, \mathrm{p}}-\mathrm{p}_{\mathrm{B}}$ overlap becomes nearly negligible: $4.0 \mathrm{kcal} / \mathrm{mol}$ (Fig. $4 D$ ).

\section{Scope of the Resistance to Oxidation of Boronic Acid-Carboxylic Acid Mixed}

Anhydrides. The oxidative stability of BOL is unique among phenylboronic acids (SI Appendix, Fig. S7). Its stability is not replicated by introducing electron-withdrawing groups on the phenyl ring or replacing the boronic acid moiety with a trifluoroborate salt. Common boronic acidprotecting groups, such as pinacol, cyclictriol, citrate, or $N$-methyliminodiacetic acid (MIDA), 
prevent complexation to intended targets while providing virtually no protection against oxidation. Even the imine adducts formed by Tris base with 2-formylphenylboronic and 2-acetylphenylboronic acid (48) are more vulnerable to oxidation than is BOL. An oxygen of an ortho nitro group has been reported to coordinate to boron $(49,50)$, and 2-nitrophenylboronic acid is more stable to oxidation than is PBA. Likewise, stability increases with electron density on the potentially coordinating oxygen of an ortho substituent containing a carbonyl group (51): formyl $<$ acetyl $<$ methoxycarbonyl $<$ aminocarbonyl. These data with coordinating oxygens are consistent with the stereoelectronic arguments put forth above. Indeed, the stereoelectronics in BOL provide more protection against oxidation than does the severe steric hindrance of two ortho methyl groups. In the end, none of the 26 other substitutions that we examined yields a phenylboronic acid with oxidative stability that is within 5\% that of BOL (Fig. S7).

Boronic Acid-Carboxylic Acid Mixed Anhydride Binds to Saccharides. PBA and BL are often employed for their ability to form esters reversibly with saccharides $(6-8,12-21)$. By using ${ }^{1} \mathrm{H}-\mathrm{NMR}$ titration, we found that BOL also forms esters with D-fructose, D-glucose, and $\mathrm{N}$-acetylneuraminic acid (Neu5Ac), though with a modest loss in affinity compared to PBA and BL (Table 2). To dissect diol-binding more thoroughly, we used the highly preorganized nopoldiol-PEG system developed by Hall and coworkers (52) along with ${ }^{1} \mathrm{H}-\mathrm{NMR}$ spectroscopy. The affinity of BOL for this ligand was higher than that of BL but lower than that of PBA (Table 2). Again, though, differences were modest. The differences in kinetics were, however, substantial. In particular, the association of BOL with nopoldiol-PEG was slow, as was its dissociation. The BOL nopoldiol-PEG complex had $t_{1 / 2}=4 \mathrm{~h}$, which exceeds by $\geq 20$-fold that of complexes formed with other boronic acids. The ability of BOL to form complexes with 1,2-diols that have high kinetic stability could be useful in applications entailing complexation to 
saccharides $(6-8,12-21)$, including the ribose moiety in nucleoside 5 '-triphosphates and at the 3' terminus of RNA.

\section{Boronic Acid-Carboxylic Acid Mixed Anhydride Acts as a Covalent Pharmacophore.}

In addition to forming reversible covalent linkages with saccharides, boronic acids can do likewise with Lewis bases in proteins. To investigate BOL in this context, we designed and synthesized stilbene $\mathbf{1}$. This compound resembles ligands that are known to bind to the tetrameric form of human transthyretin and thereby deter its aggregation into fibrils that underlie human amyloidogenic diseases $(53,54)$. We found that stilbene 1 attenuates the formation of transthyretin fibrils in a concentration-dependent manner (SI Appendix, Table S14). Then, we used X-ray crystallography to determine the three-dimensional structure of the transthyretin·stilbene 1 complex. We observed that stilbene $\mathbf{1}$ interacts covalently with transthyretin, forming a boronate ester with a serine side chain (Fig. 6 and SI Appendix, Fig. S11). Reversible covalent inhibitors like stilbene 1 can accrue benefits from covalency while avoiding the risks of permanent "off-target" modifications $(55,56)$.

Conclusions. We have discovered that the formation of a mixed anhydride with a carboxylic acid greatly increases the oxidative stability of boronic acids. The endowed stability results from stereoelectronic constraints imposed by the ring through competing donor-acceptor interactions. The ensuing "twisting" and "pulling" diminishes stabilization of the p orbital that develops on boron in the rate-limiting step for oxidation. The oxaborolones maintain the signature ability of boronic acids to form reversible covalent linkages with diols (including saccharides) and proteins. They could also act as bioisosteres for other functional groups $(57,58)$. These findings suggest that oxaborolones could be of utility in chemical biology, medicinal chemistry, and related venues. 


\section{Materials and Methods}

General. Reagents were obtained from commercial sources and used without further purification. Boronic acids were from Combi-Blocks except for phenylboronic acid (PBA) and 2-hydroxymethylphenylboronic acid (2-HMPBA), which were from Sigma-Aldrich. The synthesis of boronic acid complexes, (-)-nopoldiol-PEG, and stilbene $\mathbf{1}$ are described in the Supporting Information. Human transthyretin was produced with recombinant DNA technology in Escherichia coli as described previously (54). Phosphate-buffered saline (PBS) was made with ingredients from Research Products International and contained (in $1.0 \mathrm{~L}$ ): $0.2 \mathrm{~g} \mathrm{KCl}, 0.2 \mathrm{~g}$ $\mathrm{KH}_{2} \mathrm{PO}_{4}, 8 \mathrm{~g} \mathrm{NaCl}$, and $2.16 \mathrm{~g} \mathrm{Na} 2 \mathrm{HPO}_{4} \cdot 7 \mathrm{H}_{2} \mathrm{O}$ at $\mathrm{pH} 7.3$.

NMR spectra were obtained with an Avance-400, Avance-500, or Avance-600 spectrometer from Bruker. The $\mathrm{pH}$ of buffers $(10 \mathrm{mM})$ was determined with an accumet XL50 $\mathrm{pH}$ meter from Fischer Scientific, calibrated with a pH 3.00 reference standard buffer from VWR International and $\mathrm{pH} 12.00,12.45$, and 13.00 reference standard buffers from Ricca Chemicals.

All procedures were performed in air at ambient temperature $\left(22{ }^{\circ} \mathrm{C}\right)$ and pressure $(1.0 \mathrm{~atm})$ unless indicated otherwise.

p $K_{\mathrm{a}}$ Determination. A $100 \mathrm{mM}$ solution of 2-CPBA was prepared in $10 \mathrm{~mL}$ of $1 \mathrm{M} \mathrm{NaOH}$ containing $\mathrm{D}_{2} \mathrm{O}(5 \% \mathrm{v} / \mathrm{v})$. The $\mathrm{pH}$ of the solution was measured and adjusted with $10 \mathrm{M} \mathrm{NaOH}$ and concentrated $\mathrm{HCl}$, and $0.65-\mathrm{mL}$ samples at known $\mathrm{pH}$ values were transferred to $5-\mathrm{mm}$ quartz NMR tubes from Norell and analyzed by ${ }^{1} \mathrm{H}$ - and ${ }^{11} \mathrm{~B}-\mathrm{NMR}$ spectroscopy, referencing to the solvent peak. For the samples in $6 \mathrm{M} \mathrm{DCl}$ in $\mathrm{D}_{2} \mathrm{O}$ and $5 \mathrm{M} \mathrm{NaOH}$ in $\mathrm{D}_{2} \mathrm{O}$, the $\mathrm{pH}$ was outside of the useful range of the $\mathrm{pH}$ probe. The acidic sample gave poor referencing from the solvent peak due to an extreme chemical shift of the water peak, and was referenced to an external 
standard of $\mathrm{BF}_{3} \cdot \mathrm{Et}_{2} \mathrm{O}$ using a sealed melting-point capillary filled with the reference compound and placed inside the NMR tube. Two distinct states were observed and were assigned to the monoanion and the dianion (SI Appendix, Fig. S1). The two peaks in the ${ }^{11} \mathrm{~B}$ spectrum were integrated, the ratio was plotted against the solution $\mathrm{pH}$, and the $\mathrm{p} K_{\mathrm{a}}$ value reported as the interpolated $\mathrm{pH}$ at which the ratio of the two states was 1:1. For 2-CMPBA, the two states could not be assigned clearly, and thus no $\mathrm{p} K_{\mathrm{a}}$ value could be determined. No peaks were observed for either boronic acid, and thus no $\mathrm{p} K_{\mathrm{a}}$ value for the transition between trigonal and tetrahedral states could be determined.

Small-Molecule Crystallography. Crystals of 2-CPBA grew out of the highly acidic conditions (6 $\mathrm{M} \mathrm{DCl}$ ) in deuterium oxide employed during NMR-titration experiments. Crystals were roughly $0.5 \mathrm{~mm} \times 0.5 \mathrm{~mm} \times 0.1 \mathrm{~mm}$. Diffraction data $(\varphi$ - and $\omega$-scans) were collected at $100 \mathrm{~K}$ on a Bruker X8 diffractometer coupled to a Bruker Photon3 CPAD detector using MoK $\alpha$ radiation $(\lambda=0.71073 \AA)$ from an $I \mu S$ microsource. Diffraction data were reduced with SAINT software from Bruker, and semi-empirical absorption correction based on equivalents was performed with the program SADABS (59). The structure was solved with SHELXT and refined with SHELXL $(60,61)$. Details regarding the structure are listed in SI Appendix, Tables S1-S7.

Chemical Kinetics. Oxidation reactions were monitored by UV absorbance using a Cary 60 UV-vis spectrophotometer from Agilent. Some extinction coefficients were determined with an M1000 plate reader from Tecan. The extinction coefficient of PBA in PBS was found to be indistinguishable in the two instruments. Buffers were degassed prior to use. Measurements were performed with 1.0-mL sample volumes in disposable, plastic, UV-transparent cuvettes. All 
measurements were performed at least in triplicate. Stocks of boronic acids and phenolates were prepared by dissolving the compounds in buffer, assisted by sonication if necessary. All stock solutions were prepared on the day of their use.

Initial wavelength scans in the range of $250-350 \mathrm{~nm}$ revealed a bathochromic shift between boronic acids and phenolate (exemplified at pH 7.4 in SI Appendix, Fig. S3). Wavelengths with optimal signal-to-noise ratios were determined from these spectra and used for singlewavelength kinetic assays. The hydrogen peroxide used as an oxidant also absorbs light at these wavelengths. Extinction coefficients for the two reactants and the product were determined at each pH (SI Appendix, Table S8). Extinction coefficients for the oxidation of boronic acids were calculated as the difference in absorbance between the product and starting materials with the equation: $\Delta \varepsilon_{\text {oxidation }}=\varepsilon_{\text {phenol }}-\left(\varepsilon_{\text {boronic acid }}+\varepsilon_{\text {hydrogen peroxide }}\right)$. Initial boronic acid concentrations were $\leq 1 \mathrm{mM}$, and the final $\mathrm{pH}$ of reaction mixtures was confirmed not to change by more than 0.1 units.

Initial velocity kinetic assays were performed by measuring the UV absorbance of $990 \mu \mathrm{L}$ of a boronic acid solution and then initiating the reaction by the addition of $10 \mu \mathrm{L}$ of a $100 \times$ hydrogen peroxide stock solution. Rates were measured in triplicate. Slopes from the first 10\% of the reaction (or from 10 min of a slow reaction: $k_{\mathrm{obs}}<10^{-2} \mathrm{M}^{-1} \mathrm{~s}^{-1}$ ) were calculated and converted to units of $\mathrm{M} / \mathrm{s}$ by dividing by the value of $\Delta \varepsilon_{\text {oxidation. }}$. Reactions were slow enough not to require a stopped-flow apparatus, but the initial velocity $\left(v_{\mathrm{o}}\right)$ for the fastest reactions $\left(k_{\mathrm{obs}} \geq\right.$ $1 \mathrm{M}^{-1} \mathrm{~S}^{-1}$ ) could only be calculated from a few dozen data points. Rates at three different concentrations of boronic acid and hydrogen peroxide were measured and determined to be firstorder with respect to both substrates (SI Appendix, Fig. S4). Second-order rate constants were determined with the equation: $k_{\mathrm{obs}}=v_{\mathrm{o}} /([$ boronic acid][hydrogen peroxide] $)$. 
Evaluation of pH-Dependence. The $\mathrm{pH}$-dependence of oxidation reactions was assessed by using the buffers listed in SI Appendix, Table S8. Reactions conducted at $\mathrm{pH}>11.7$ (which is the $\mathrm{p} K_{\mathrm{a}}$ of hydrogen peroxide) engendered the evolution of gas. These rates, which were slower than expected, were omitted from the analysis, though the relative rates of the different boronic acids at $\mathrm{pH}>11.7$ fitted the expected trend.

Competition Assay. Initial velocity kinetics were performed by monitoring the oxidation of coumarin boronic acid (CBA) by hydrogen peroxide in PBS. The production of 7-hydroxycoumarin at ambient temperature was monitored by measuring the increase in fluorescence emission at $450 \mathrm{~nm}$ following excitation at $365 \mathrm{~nm}$ using a Quantamaster spectrophotometer from Photon Technologies International. Slopes for the oxidation of $500 \mathrm{nM}$ CBA were confirmed to be linear from 5-500 $\mu \mathrm{M}$ hydrogen peroxide, thus providing a useful range in which a reduction in velocity provides a metric to assess the formation of a competitive complex formed between hydrogen peroxide and 2-CPBA. Oxidation of $500 \mathrm{nM}$ CBA reached $10 \%$ completion after roughly $100 \mathrm{~s}$, in which time negligible amounts of 2-CPBA would be oxidized. CBA oxidations were performed with $0.50 \mu \mathrm{M}$ CBA in the presence of $2-\mathrm{CPBA}(0-$ $250 \mathrm{mM})$ and hydrogen peroxide $(0.50 \mathrm{mM})$. Values of $\partial$ fluorescence $/ \partial t$ were obtained by linear regression analysis, normalized to the value with $0 \mathrm{mM} 2-\mathrm{CPBA}$, and plotted as a function of 2-CPBA concentration using Prism 8 software from GraphPad.

Computational Analyses. Structure optimizations were performed using Gaussian 16 software from Gaussian at the M06-2X/6-311+G(d,p) level of theory and employing the IEFPCM 
solvation model for water (62-64). Frequency calculations were performed to confirm each stationary point as a minimum or first-order saddle point. Rotamers about the $\mathrm{B}-\mathrm{O}$ and $\mathrm{O}-\mathrm{H}$ bonds were examined to reveal the global minimum-energy conformation. Energies for those conformations and imaginary frequencies of transition states are listed in SI Appendix, Table S10. Coordinates are listed in the Supporting Information.

Natural Bonding Orbital (NBO) analysis of the optimized structures were conducted with NBO 6.0 software (47). Interaction energies, PNBO overlap integrals (S), and the off-diagonal NBO Fock Matrix elements (which are proportional to the overlap integrals) are listed in $S I$ Appendix, Table S13. The NBO "\$CHOOSE" keyword was used to give the desired Lewis structure for the BL and BON transition states.

Binding to Saccharides. The affinity of boronic acids for saccharides was evaluated by using ${ }^{1} \mathrm{H}-\mathrm{NMR}$ spectroscopy to determine the ratio of complexed to uncomplexed boronic acid, as described previously (65). Due to its low affinity, D-glucose was used at twice the concentrations of other saccharides. Representative ${ }^{1} \mathrm{H}-\mathrm{NMR}$ spectra are shown in SI Appendix, Fig. S8.

Binding to (-)-Nopoldiol-PEG. Measurements of $k_{\text {on }}$ and $K_{\text {eq }}$ for the binding of boronic acids to (-)-nopoldiol-PEG was performed as described previously (52). Representative ${ }^{1} \mathrm{H}-\mathrm{NMR}$ spectra are shown in SI Appendix, Fig. S9 and S10.

Inhibition of Fibril Formation. Transthyretin fibril formation and the inhibition thereof was assessed with light scattering, as described previously $(54,66)$. Data are listed in Table S14. 
Protein Crystallography. Protein stocks of transthyretin were concentrated to $6.0 \mathrm{mg} / \mathrm{mL}$ in $10 \mathrm{mM}$ sodium phosphate buffer, $\mathrm{pH}$ 7.6, containing $\mathrm{KCl}(100 \mathrm{mM})$. Transthyretin stocks were incubated with a 10-fold excess of benzoxaborolone $\mathbf{1}$ for $30 \mathrm{~min}$ at room temperature. Crystals of transthyretin were obtained by hanging-drop vapor diffusion by twofold dilution of complex stocks with mother liquor containing 1.3 M sodium citrate buffer, $\mathrm{pH}$ 5.5, containing glycerol $(2 \% \mathrm{v} / \mathrm{v})$ to form $4-\mu \mathrm{L}$ drops. Crystals grew within $24 \mathrm{~h}$. Crystals were cryo-protected with a solution of 1.5 M sodium citrate buffer, $\mathrm{pH} 5.5$, containing glycerol (10\% v/v). Single-crystal X-ray diffraction experiments were performed with a Bruker X8 diffractometer equipped with a Bruker Photon3 detector using $\mathrm{CuK} \alpha$ radiation $(\lambda=1.54178 \AA)$ from a $I \mu S$ source. Data were indexed with APEX3 software, reduced with SAINT, and scaled and absorption corrected using SADABS (59). Data collection statistics are listed in SI Appendix, Table S15.

The atomic structure of the transthyretin·stilbene 1 complex was solved by molecular replacement using the Phaser program as implemented in PHENIX and employing PDB entry $5 \mathrm{u} 4 \mathrm{f}(54)$ as a search model. Iterative refinement and model building was performed with phenix.refine, and COOT $(67,68)$. Densities were observed in the T4-binding pockets. Restraints for stilbene 1, with both borolone moieties in the tetrahedral state, were generated with eLBOW and the ligand was placed with COOT. There was insufficient density for both hydroxy groups for the boron in the interior of the pocket, and they were removed for subsequent refinement. In the ensuing model, the hydroxy group of an alternative conformation of Ser117 was found to be co-planar with the interior benzoxaborolone ring, indicative of the formation of a trigonal ester. Additional restraints were imposed to provide accurate bond distances and angles, as described previously (54). 


\section{Acknowledgements}

We are grateful to Dr. Peter Müller (Department of Chemistry, Massachusetts Institute of Technology) for determining the crystal structure of 2-CPBA. B. G. was supported by an Arnold O. Beckman Postdoctoral Fellowship. This work was supported by grant R01 GM044783 (NIH). This work used the Extreme Science and Engineering Discovery Environment (XSEDE), which is supported by the National Science Foundation grant number ACI-1548562. Specifically, it used the Bridges system, which is supported by NSF award number ACI-1445606, at the Pittsburgh Supercomputing Center (PSC). This work also used the UNM Center for Advanced Research Computing, supported in part by the National Science Foundation.

\section{Conflict of interest}

The authors declare no conflict of interest. 
1. G. W. Bemis, M. A. Murcko, The properties of known drugs. 1. Molecular frameworks. J. Med. Chem. 39, 2887-2893 (1996).

2. G. W. Bemis, M. A. Murcko, Properties of known drugs. 2. Side chains. J. Med. Chem. 42, 5095-5099 (1999).

3. S. J. Baker, C. Z. Ding, Y.-K. Zhang, V. Hernandez, Y. Xia, Therapeutic potential of boroncontaining compounds. Future Med. Chem. 1, 1275-1288 (2009).

4. G. F. S. Fernandes, W. A. Denny, J. L. Dos Santos, Boron in drug design: Recent advances in the development of new therapeutic agents. Eur. J. Med. Chem. 179, 791-804 (2019).

5. B. Muz et al., Spotlight on ixazomib: Potential in the treatment of multiple myeloma. Drug Des. Devel. Ther. 10, 217-226 (2016).

6. M. Dowlut, D. G. Hall, An improved class of sugar-binding boronic acids, soluble and capable of complexing glycosides in neutral water. J. Am. Chem. Soc. 128, 4226-4227 (2006).

7. A. Adamczyk-Woźniak, M. K. Cyrański, A. Żubrowska, A. Sporzyński, Benzoxaboroles-Old compounds with new applications. J. Organomet. Chem. 694, 3533-3541 (2009).

8. A. Adamczyk-Woźniak, K. M. Borys, A. Sporzyński, Recent developments in the chemistry and biological applications of benzoxaboroles. Chem. Rev. 115, 5224-5247 (2015).

9. C. T. Liu, J. W. Tomsho, S. J. Benkovic, The unique chemistry of benzoxaboroles: Current and emerging applications in biotechnology and therapeutic treatments. Bioorg. Med. Chem. 22, 4462-4473 (2014).

10. S. J. Hecker et al., Discovery of a cyclic boronic acid $\beta$-lactamase inhibitor (RPX7009) with utility vs Class A serine carbapenemases. J. Med. Chem. 58, 3682-3691 (2015).

11. E. D. Farfán-García et al., Current data regarding the structure-toxicity relationship of boroncontaining compounds. Toxicol. Lett. 258, 115-125 (2016).

12. K. Severin, Boronic acids as building blocks for molecular nanostructures and polymeric materials. Dalton Trans., 5254-5264 (2009). 
13. D. G. Hall, Boronic Acids: Preparation and Applications in Organic Synthesis, Medicine and Materials, $2^{\text {nd }}$ ed. (Wiley-VCH, Weinheim, Germany, 2011).

14. G. F. Whyte, R. Vilar, R. Woscholski, Molecular recognition with boronic acids-applications in chemical biology. J. Chem. Biol. 6, 161-174 (2013).

15. B. C. Das et al., Boron chemicals in diagnosis and therapeutics. Future Med. Chem. 5, 653676 (2013).

16. C. Zhu, J. R. Falck, Transition-metal-free ipso-functionalizaiton of arylboronic acids and derivatives. Adv. Synth. Catal. 356, 2395-2410 (2014).

17. W. L. A. Brooks, B. S. Sumerlin, Synthesis and applications of boronic acid-containing polymers: From materials to medicine. Chem. Rev. 116, 1375-1397 (2015).

18. D. Li, Y. Chen, Z. Liu, Boronate affinity materials for separation and molecular recognition: Structure, properties and applications. Chem. Soc. Rev. 44, 8097-8123 (2015).

19. D. B. Diaz, A. K. Yudin, The versatility of boron in biological target engagement. Nat. Chem. 9, 731-742 (2017).

20. S. Cambray, J. Gao, Versatile bioconjugation chemistries of ortho-boronyl aryl ketones and aldehydes. Acc. Chem. Res. 51, 2198-2206 (2018).

21. D. G. Hall, B. Akgun, Boronic acids as bioorthogonal probes in site-selective labeling of proteins. Angew. Chem. Int. Ed. 57, 13028-13044 (2018).

22. J. P. M. António, R. Russo, C. P. Carvalho, P. M. S. D. Cal, P. M. P. Gois, Boronic acids as building blocks for the construction of therapeutically useful bioconjugates. Chem. Soc. Rev. 48, 3513-3536 (2019).

23. R. T. Jacobs et al., SCYX-7158, an orally-active benzoxaborole for the treatment of stage 2 human African trypanosomiasis. PLoS Negl. Trop. Dis. 5, e1151 (2011).

24. W. Bu et al., Early rapid identification of in vivo rat metabolites of AN6414, a novel boroncontaining PDE4 inhibitor by QTRAP LC/MS/MS to support drug discovery. J. Pharm. Biomed. Anal. 70, 344-353 (2012). 
25. B. R. Caes, M. J. Palte, R. T. Raines, Organocatalytic conversion of cellulose into a platform chemical. Chem. Sci. 4, 196-199 (2013).

26. T. Hashimoto, A. O. Gálvez, K. Maruoka, In situ assembled boronate ester assisted chiral carboxylic acid catalyzed asymmetric trans-aziridinations. J. Am. Chem. Soc. 135, 1766717670 (2013).

27. B. J. Graham, R. T. Raines, Efficient metal-free conversion of glucose to 5hydroxymethylfurfural using a boronic acid. Biomass Conv. Bioref. 9, 471-477 (2019).

28. S. Vshyvenko, M. L. Clapson, I. Suzuki, D. G. Hall, Characterization of the dynamic equilibrium between closed and open forms of the benzoxaborole pharmacophore. ACS Med. Chem. Lett. 7, 1097-1101 (2016).

29. A. D. Ainley, F. Challenger, CCLXXX.-Studies of the boron-carbon linkage. Part I. The oxidation and nitration of phenylboric acid. J. Chem. Soc., 2171-2180 (1930).

30. H. G. Kuivila, Electrophilic displacement reactions. III. Kinetics of the reaction between hydrogen peroxide and benzeneboronic acid. J. Am. Chem. Soc. 76, 870-874 (1954).

31. M. Hawthorne, Simple procedure for the conversion of aryl halides to the corresponding phenols. J. Org. Chem. 22, 1001-1001 (1957).

32. H. G. Kuivila, A. G. Armour, Electrophilic displacement reactions. IX. Effects of substituents on rates of reactions between hydrogen peroxide and benzeneboronic acid. J. Am. Chem. Soc. $79,5659-5662$ (1957).

33. M. Giorgio, M. Trinei, E. Migliaccio, P. G. Pelicci, Hydrogen peroxide: A metabolic byproduct or a common mediator of ageing signals? Nat. Rev. Mol. Cell Biol. 8, 722-728 (2007).

34. A. R. Lippert, G. C. Van de Bittner, C. J. Chang, Boronate oxidation as a bioorthogonal reaction approach for studying the chemistry of hydrogen peroxide in living systems. Acc. Chem. Res. 44, 793-804 (2011). 
35. Q. Zhong et al., Boronic prodrug of 4-hydroxytamoxifen is more efficacious than tamoxifen with enhanced bioavailability independent of CYP2D6 status. BMC Cancer 15, 625 (2015).

36. T. T. Hoang, T. P. Smith, R. T. Raines, A boronic acid conjugate of angiogenin that shows ROS-responsive neuroprotective activity. Angew. Chem. Int. Ed. 56, 2619-2622 (2017).

37. H. Decker, K. E. van Holde, Oxygen and the Evolution of Life (Springer, New York, NY, 2011).

38. H. Sies, C. Berndt, D. P. Jones, Oxidative stress. Annu. Rev. Biochem. 86, 715-748 (2017).

39. A. Sikora, J. Zielonka, M. Lopez, J. Joseph, B. Kalyanaraman, Direct oxidation of boronates by peroxynitrite: Mechanism and implications in fluorescence imaging of peroxynitrite. Free Radic. Biol. Med. 47, 1401-1407 (2009).

40. J. Zielonka et al., Boronate probes as diagnostic tools for real time monitoring of peroxynitrite and hydroperoxides. Chem. Res. Toxicol. 25, 1793-1799 (2012).

41. T. Matsumura, S. Iwatsuki, K. Ishihara, Direct kinetic measurements for the fast interconversion process between trigonal boronic acid and tetragonal boronate ion at low temperatures. Inorg. Chem. Commun. 8, 713-716 (2005).

42. Y. Yamamoto et al., Fast trigonal/tetragonal interconversion followed by slow chelate-ring closure in the complexation of boronic acids. Inorg. Chim. Acta 358, 3355-3361 (2005).

43. N. Ni et al., Probing the general time scale question of boronic acid binding with sugars in aqueous solution at physiological pH. Bioorg. Med. Chem. 20, 2957-2961 (2012).

44. J. D. Larkin, G. D. Markham, M. Milkevitch, B. R. Brooks, C. W. Bock, Computational investigation of the oxidative deboronation of boroglycine, $\mathrm{H}_{2} \mathrm{~N}-\mathrm{CH}_{2}-\mathrm{B}(\mathrm{OH})_{2}$, Using $\mathrm{H}_{2} \mathrm{O}$ and $\mathrm{H}_{2} \mathrm{O}_{2}$. J. Phys. Chem. A 113, 11028-11034 (2009).

45. Y. Q. Zou et al., Highly efficient aerobic oxidative hydroxylation of arylboronic acids: Photoredox catalysis using visible light. Angew. Chem. Int. Ed. 51, 784-788 (2012). 
46. T. Toyao et al., Visible-light, photoredox catalyzed, oxidative hydroxylation of arylboronic acids using a metal-organic framework containing tetrakis(carboxyphenyl)porphyrin groups. Chem. Commun. 51, 16103-16106 (2015).

47. E. D. Glendening et al., NBO 6.0 (Theoretical Chemistry Institute, University of Wisconsin, Madison, WI, 2013).

48. K. Li, M. A. Kelly, J. Gao, Biocompatible conjugation of Tris base to 2-acetyl and 2-formyl phenylboronic acid. Org. Biomol. Chem. 17, 5908-5912 (2019).

49. B. Bettman, G. E. K. Branch, D. L. Yarbroff, Dissociation constants of organic boric acids. J. Am. Chem. Soc. 56, 1865-1870 (1934).

50. S. Soundararajan, E. N. Duesler, J. H. Hageman, Structure of 4-carboxy-2nitrobenzeneboronic acid. Acta Crystallogr. C49, 690-693 (1993).

51. S. Toyota, M. Asakura, T. Sakaue, Intramolecular $C=O \cdots B$ interactions in o-boron substituted benzaldehyde, acetophenone, and benzophenone. Bull. Chem. Soc. Jpn. 75, 2667-2671 (2002).

52. B. Akgun, D. G. Hall, Fast and tight boronate formation for click bioorthogonal conjugation. Angew. Chem. Int. Ed. 55, 3909-3913 (2016).

53. S. M. Johnson et al., Native state kinetic stabilization as a strategy to ameliorate protein misfolding diseases: A focus on the transthyretin amyloidoses. Acc. Chem. Res. 38, 911921 (2005).

54. T. P. Smith, I. W. Windsor, K. T. Forest, R. T. Raines, Stilbene boronic acids form a covalent bond with human transthyretin and inhibit its aggregation. J. Med. Chem. 60, 7820-7834 (2017).

55. A. Bandyopadhyay, J. Gao, Targeting biomolecules with reversible covalent chemistry. Curr. Opin. Chem. Biol. 34, 110-116 (2016).

56. T. A. Baillie, Approaches to mitigate the risk of serious adverse reactions in covalent drug design. Expert Opin. Drug. Discov., DOI:10.1080/17460441.17462021.11832079 (2020). 
57. N. Brown, Ed., Bioisosteres in Medicinal Chemistry (Wiley-VCH, Weinheim, Germany, 2012).

58. I. W. Windsor et al., Sub-picomolar inhibition of HIV-1 protease with a boronic acid. J. Am. Chem. Soc. 140, 14015-14018 (2018).

59. L. Krause, R. Herbst-Irmer, G. M. Sheldrick, D. Stalke, Comparison of silver and molybdenum microfocus X-ray sources for single-crystal structure determination. J. Appl. Crystallogr. 48, 3-10 (2015).

60. G. Sheldrick, A short history of SHELX. Acta Crystallogr. A64, 112-122 (2008).

61. G. Sheldrick, SHELXT_-Integrated space-group and crystal-structure determination. Acta Crystallogr. A71, 3-8 (2015).

62. M. J. Frisch et al., Gaussian 16, Revision A.03 (Gaussian, Inc., Wallingford CT, 2016).

63. N. Mardirossian, M. Head-Gordon, How accurate are the Minnesota density functionals for noncovalent interactions, isomerization energies, thermochemistry, and barrier heights involving molecules composed of main-group elements? J. Chem. Theory Comput. 12, 4303-4325 (2016).

64. Y. Wang, X. Jin, H. S. Yu, D. G. Truhlar, X. He, Revised M06-L functional for improved accuracy on chemical reaction barrier heights, noncovalent interactions, and solid-state physics. Proc. Natl. Acad. Sci. USA 114, 8487-8492 (2017).

65. G. A. Ellis, M. J. Palte, R. T. Raines, Boronate-mediated biologic delivery. J. Am. Chem. Soc. 134, 3631-3634 (2012).

66. G. J. Miroy et al., Inhibiting transthyretin amyloid fibril formation via protein stabilization. Proc. Natl. Acad. Sci. USA 93, 15051-15056 (1996).

67. P. D. Adams et al., PHENIX: A comprehensive Python-based system for macromolecular structure solution. Acta Crystallogr. D66, 213-221 (2010).

68. P. Emsley, B. Lohkamp, W. G. Scott, K. Cowtan, Features and development of COOT. Acta Crystallogr. D66, 486-501 (2010). 
69. C. C. Winterbourn, D. Metodiewa, Reactivity of biologically important thiol compounds with superoxide and hydrogen peroxide. Free Radic. Biol. Med. 27, 322-328 (1999).

70. J. W. Tomsho, A. Pal, D. G. Hall, S. J. Benkovic, Ring structure and aromatic substituent effects on the $\mathrm{pK}_{\mathrm{a}}$ of the benzoxaborole pharmacophore. ACS Med. Chem. Lett. 3, 48-52 (2012).

71. L. I. Bosch, T. M. Fyles, T. D. James, Binary and ternary phenylboronic acid complexes with saccharides and Lewis bases. Tetrahedron 60, 11175-11190 (2004). 
Table 1. Second-Order Rate Constants for the Oxidation of Boronic Acids and Biological Thiols by Hydrogen Peroxide at Physiological $\mathrm{pH}$.

\begin{tabular}{lll}
\hline Compound & $k_{\text {obs }}\left(\mathrm{M}^{-1} \mathrm{~s}^{-1}\right)$ & $k_{\mathrm{obs}}(\mathrm{PBA}) / k_{\mathrm{obs}}$ \\
\hline $\mathrm{PBA}$ & 2.4 & 1 \\
$\mathrm{BL}$ & 2.4 & 1.0 \\
$\mathrm{BN}$ & 8.1 & 0.30 \\
$\mathrm{BON}$ & 0.041 & $5.9 \times 10^{1}$ \\
$\mathrm{BOL}$ & 0.00015 & $1.6 \times 10^{4}$ \\
Cysteine $^{a}$ & 2.9 & 0.83 \\
Cysteamine $^{a}$ & 2.9 & 0.83 \\
N-Acetylcysteine $^{a}$ & 0.16 & $1.5 \times 10^{1}$ \\
Glutathione $^{a}$ & 0.87 & 2.8 \\
\hline
\end{tabular}

${ }^{a}$ Values are from ref. 69. 
Table 2. Thermodynamic and Kinetic Parameters for Boronic Acid Oxygen-Exchange and Binding to Saccharides and Nopoldiol-PEG

\begin{tabular}{|c|c|c|c|c|c|c|}
\hline \multirow[b]{2}{*}{$\begin{array}{c}\text { Boronic } \\
\text { Acid }\end{array}$} & \multicolumn{3}{|c|}{$\begin{array}{c}\text { Saccharide binding } \\
K_{a}\left(\mathrm{M}^{-1}\right)^{a}\end{array}$} & \multicolumn{3}{|c|}{ Nopoldiol-PEG binding } \\
\hline & D-fructose & D-glucose & Neu5Ac & $K_{a}\left(\mathrm{M}^{-1}\right)^{b}$ & $k_{\text {on }}\left(\mathrm{M}^{-1} \mathrm{~s}^{-1}\right)^{c}$ & $k_{\text {off }}\left(\mathrm{s}^{-1}\right)^{d}$ \\
\hline PBA & $128 \pm 20$ & $5 \pm 1$ & $13 \pm 1$ & $3000 \pm 1600$ & $3.4 \pm 1.8$ & $(1.1 \pm 0.9) \times 10^{-3}$ \\
\hline$B L$ & $336 \pm 43$ & $28 \pm 4$ & $43 \pm 5$ & $550 \pm 20$ & $>50$ & $>(9.1 \pm 0.3) \times 10^{-2}$ \\
\hline $\mathrm{BOL}$ & $23 \pm 2$ & $1.7 \pm 0.1$ & $6.0 \pm 0.5$ & $2300 \pm 380$ & $0.12 \pm 0.05$ & $(5.2 \pm 2.3) \times 10^{-5}$ \\
\hline
\end{tabular}

${ }^{a}$ Values for $\mathrm{BOL}$ are the mean $\pm \mathrm{SD}$ of at least 5 independent measurements (SI Appendix, Fig. S8). Values for PBA and BL are from ref. $65 .{ }^{b}$ Mean \pm SD of at least 4 independent measurements. 'Values are the mean \pm SD of 3 independent measurements (SI Appendix, Fig. S9 and S10). The value of $k_{\text {on }}$ for $\mathrm{BL}$ was too large to be determined with ${ }^{1} \mathrm{H}-\mathrm{NMR}$ spectroscopy. ${ }^{d}$ Calculated as $k_{\text {off }}=k_{\text {on }} / K_{\text {a }}$. 


\section{FIGURE LEGENDS}

Fig. 1. (A) FDA-approved pharmaceuticals containing a boronic acid. (B) Putative mechanism for the oxidative deboronation of a boronic acid by hydrogen peroxide (30).

Fig. 2. (A) Structures of relevant hydration and protonation states, and $p K_{a}$ values of benzoxaborinine (2-HEPBA: BN) (70), phenylboronic acid (PBA) (71), benzoxaborole (2-HMPBA; BL) (70), benzoxaborininone (2-CMPBA; BON), and benzoxaborolone (2-CPBA; BOL) (this work). Inset: ORTEP diagram of hydrated BOL from X-ray crystallography. $(B)$ $\mathrm{pH}$-Dependence of the observed second-order rate constant for the oxidation of boronic acids by hydrogen peroxide. Data were fitted to the equation $k_{\mathrm{obs}}=k_{1}+k_{2} /\left(1+10^{\mathrm{pKa}-\mathrm{pH}}\right)$ by using p $K_{\mathrm{a}}$ values in panel $A$ to give $k_{1}=(3.2 \pm 0.6) \times 10^{-3} \mathrm{M}^{-1} \mathrm{~s}^{-1}, k_{2}=59 \pm 5 \mathrm{M}^{-1} \mathrm{~s}^{-1}$ for $\mathrm{BN} ; k_{1}=(1.1 \pm 0.2)$ $\times 10^{-2} \mathrm{M}^{-1} \mathrm{~s}^{-1}, k_{2}=49 \pm 4 \mathrm{M}^{-1} \mathrm{~s}^{-1}$ for PBA; $k_{1}=(3.4 \pm 0.4) \times 10^{-3} \mathrm{M}^{-1} \mathrm{~s}^{-1}, k_{2}=4.2 \pm 0.2 \mathrm{M}^{-1} \mathrm{~s}^{-1}$ for $\mathrm{BL} ;$ and $k_{1}=(1.6 \pm 0.1) \times 10^{-4} \mathrm{M}^{-1} \mathrm{~s}^{-1}, k_{2}=9.7 \pm 1.3 \mathrm{M}^{-1} \mathrm{~s}^{-1}$ for BOL. Data for BON show no $\mathrm{pH}$-dependence with a mean $k_{\mathrm{obs}}=0.041 \pm 0.017 \mathrm{M}^{-1} \mathrm{~s}^{-1}$.

Fig. 3. Inhibition of the oxidation of coumarin boronic acid by BOL. $(A)$ Scheme depicting the competition assay. $(B)$ Plot of initial rates of the oxidation of CBA $(0.50 \mu M)$ by $\mathrm{H}_{2} \mathrm{O}_{2}(0.50 \mathrm{mM})$ in PBS in the presence of BOL (0-250 mM). Data are derived from the fluorescence of 7-hydroxycoumarin.

Fig. 4. Structures of key overlapping orbitals in the transition state for the $B \rightarrow O$ shift during the oxidation of $(A)$ PBA, $(B) \mathrm{BL},(C) \mathrm{BON}$, and $(D) \mathrm{BOL}$. As calculated at the M06-2X/6-311+G(d,p) level of theory. Images were created with NBOView 1.1. Numerical data are the second-order perturbation energies $\left(E^{(2)}\right)$ and PNBO overlap integrals $(S)$ for the $\mathrm{n}_{\mathrm{O}, \mathrm{p}} \rightarrow \mathrm{p}_{\mathrm{B}}$ interaction in the transition states. Additional computational data are listed in Table S13. 
Fig. 5. Graph of experimental and calculated relative rate constants for the oxidation of the monoanionic forms of PBA, BL, BON, and BOL by hydrogen peroxide. Images show the calculated structure of the transition states for the rate-limiting 1,2-aryl shift during the oxidation reaction. Structures were optimized at the M06-2X/6-311+G(d,p) level of theory using the IEFPCM solvation model. Values of $k_{\text {monoanion }}$ are listed in Table S12.

Fig. 6. Crystal structure of stilbene 1 bound to human transthyretin, showing the formation of a boronate ester with Ser117 (PDB entry 6u0q). 
Fig. 1
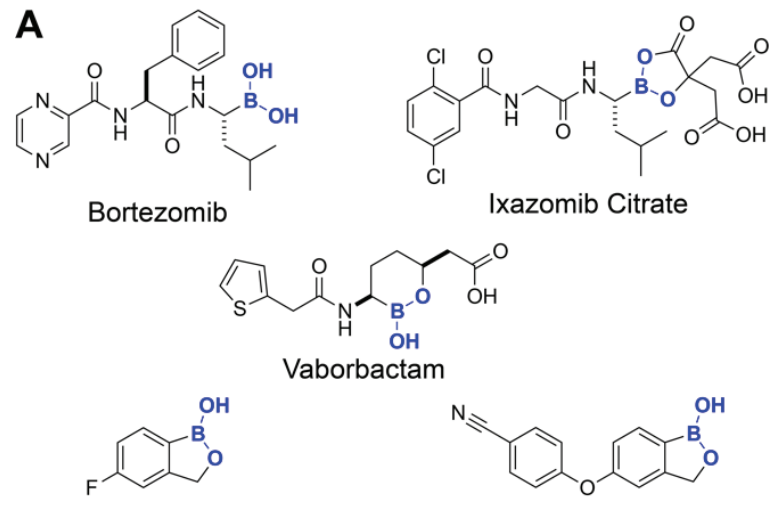

Tavaborole

Crisaborole

B

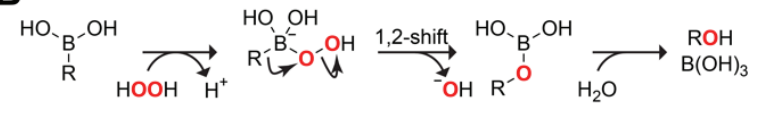


Fig. 2

A
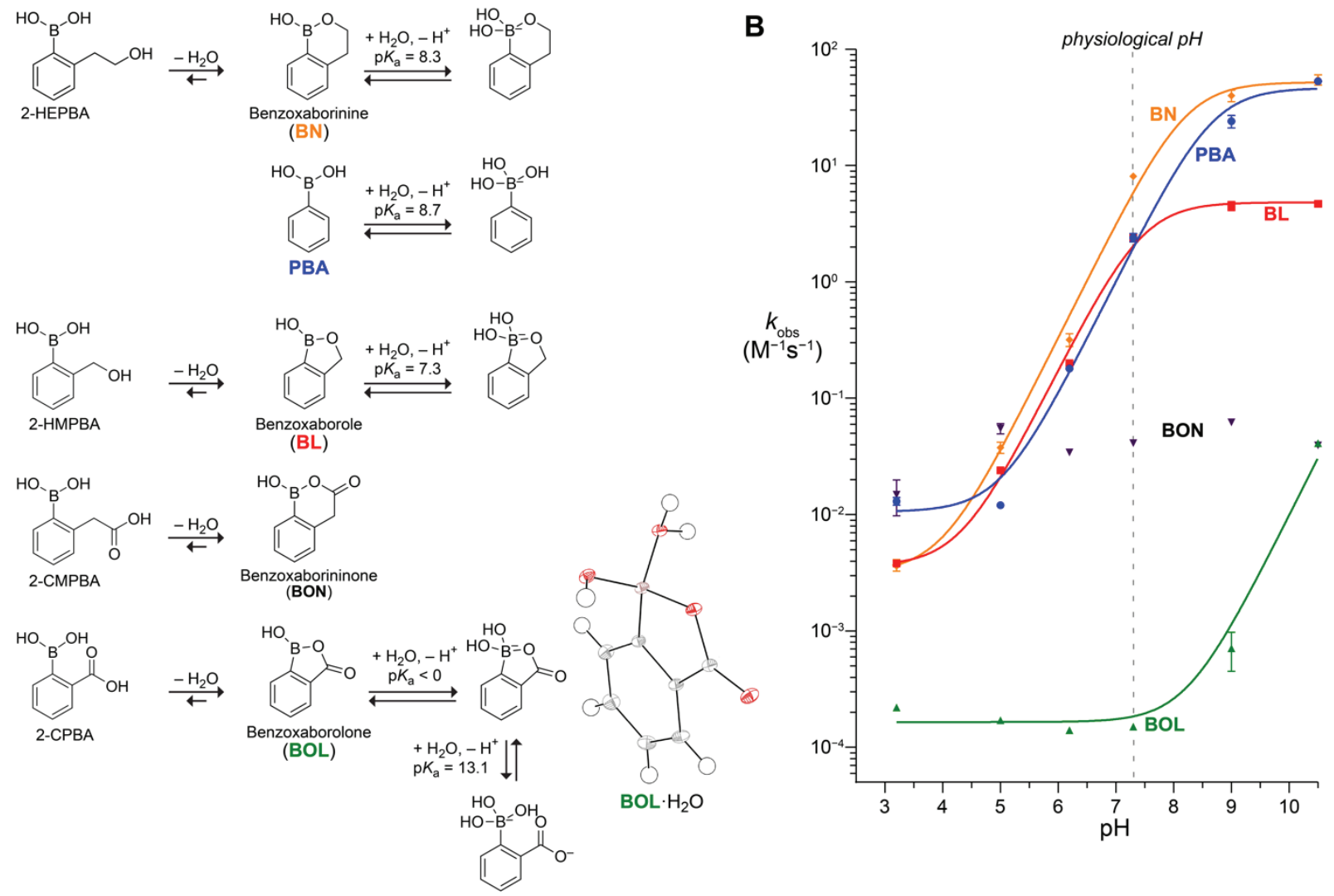
Fig. 3
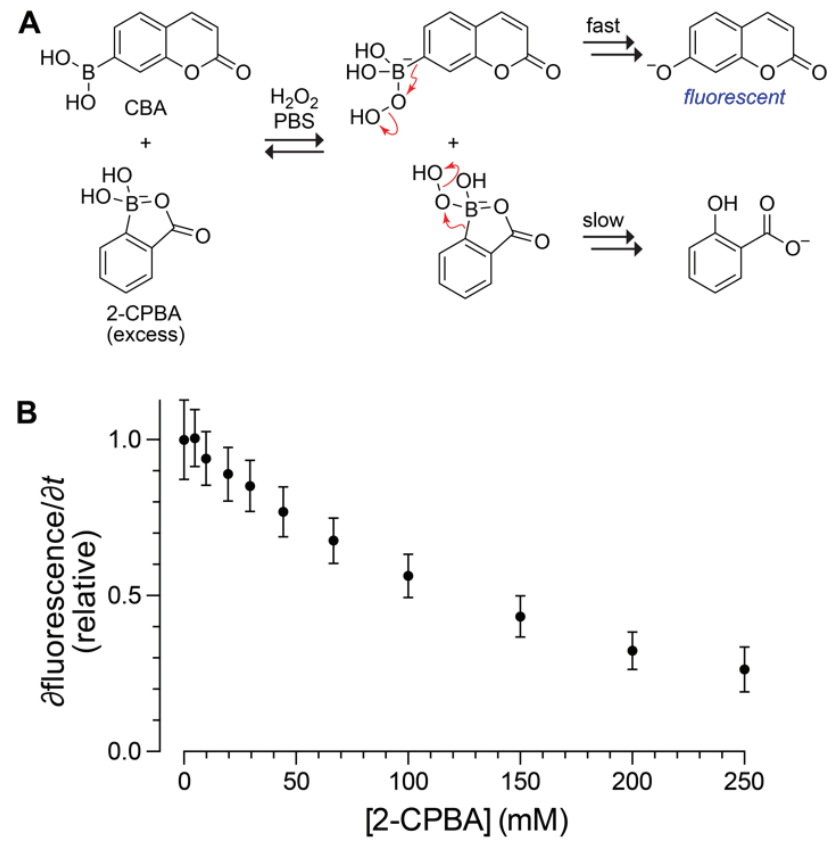
Fig. 4
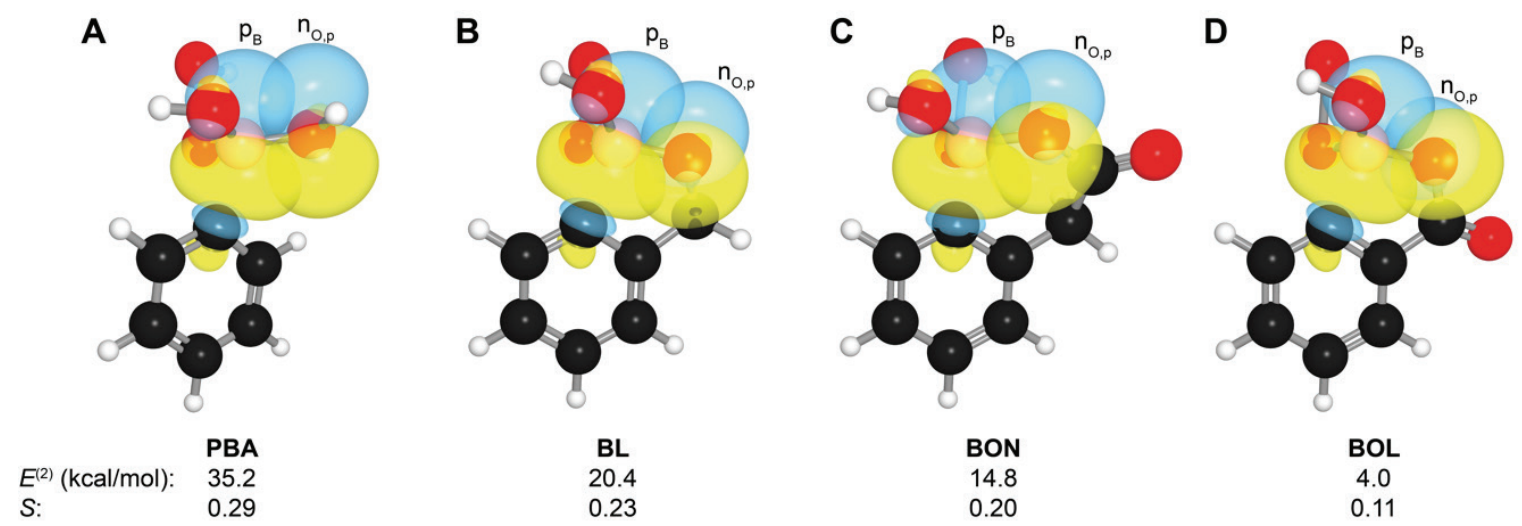
Fig. 5

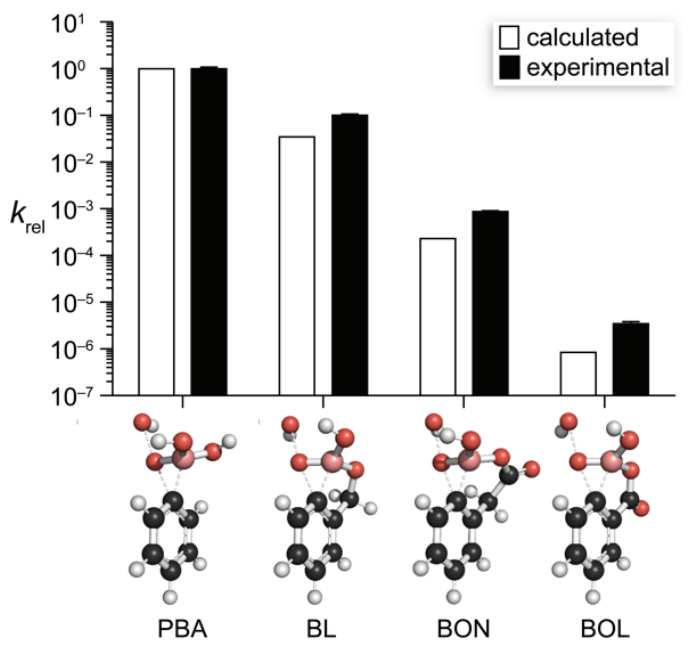


Fig. 6
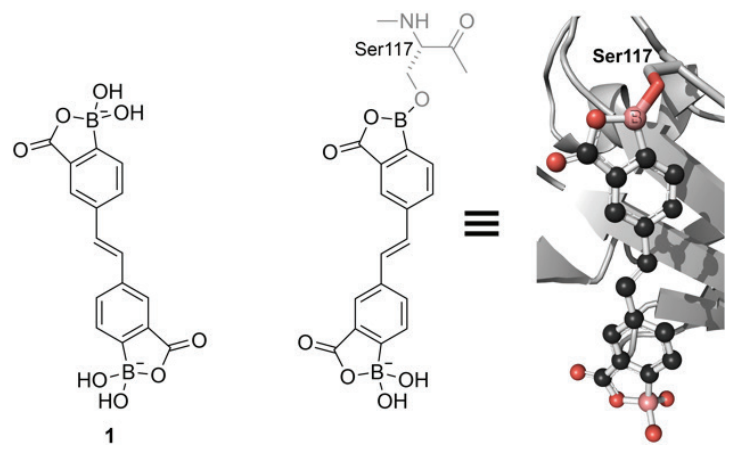